\title{
PET and Neck Ultrasound for the Detection of Cervical Lymphadenopathy in Patients with Lung Cancer and Mediastinal Lymphadenopathy
}

\author{
Mohammed Ahmed ${ }^{a} \quad$ Antoinette Flannery $^{a} \quad$ Cyrus Daneshvar $^{b} \quad$ David Breen $^{a}$ \\ a Interventional Respiratory Unit, Galway University Hospital, Galway, Ireland; b Department of Respiratory Medicine, \\ Plymouth Hospitals NHS Trust, Plymouth, UK
}

\section{Keywords}

Lung cancer · Cervical lymphadenopathy · Ultrasound .

Positron emission tomography

\begin{abstract}
Background: Cervical lymph nodes are frequently involved in patients with lung cancer and indicate inoperability. Some guidelines recommend neck ultrasound (NUS) in patients with bulky mediastinal lymphadenopathy. Positron emission tomography (PET) is indicated for patients with potentially curable disease. Objectives: We aimed to assess the diagnostic yield of NUS and the diagnostic accuracy of PET for cervical lymphadenopathy in this group with a high pre-test probability of N3 disease. Methods: Records of all patients with lung cancer who underwent an NUS over a consecutive 5 -year period were reviewed. Only patients with mediastinal lymphadenopathy on computerised tomography (CT) were included. The diagnostic accuracy of PET was assessed with NUS-guided fine needle aspiration cytology used as the reference test. Results: During the study period, 123 patients met the inclusion criteria. Malignant cervical lymphadenopathy was confirmed in 49/123 (39.8\% [95\% Cl 31.1-49.1]). PET-CT had a specificity of $81.1 \%$, sensitivity of $87.5 \%$, negative predictive value of $96.8 \%$ and positive predictive value of $50 \%$ for the detection of cervical lymphadenopathy, and
\end{abstract}

\section{KARGER}

(c) 2018 S. Karger AG, Basel

E-Mail karger@karger.com

www.karger.com/res it contributed no additional staging information in the neck area. Overall, PET led to a change in management in only $2.2 \%$ of cases. Conclusion: A significant proportion of patients with lung cancer and mediastinal lymphadenopathy have cervical lymphadenopathy detected by NUS. In this group of patients, PET offers minimal additional value in staging and management.

(c) 2018 S. Karger AG, Basel

\section{Introduction}

Patients with lung cancer frequently present at an advanced stage [1]. Decisions regarding treatment depends on accurate staging that should be performed using non-invasive investigations followed by selection of suitable sites for tissue sampling. Presence of lower cervical lymphadenopathy indicates N3 disease corresponding to at least stage IIIB, and upper cervical lymphadenopathy represents M1b disease - stage IV lung cancer [2]. Neck ultrasound (NUS) routinely performed in patients with mediastinal lymphadenopathy has been shown to reveal metastases to neck lymph nodes in 40 $50 \%$ of cases $[3,4]$.

Positron emission tomography (PET) is indicated when computerised tomography $(\mathrm{CT})$ of the thorax and 
upper abdomen shows evidence of potentially curable disease. For staging of the mediastinum, PET-CT consistently shows a significant rate of false-positive findings. A systematic review of 45 studies on the diagnostic accuracy of PET for mediastinal lymphadenopathy in lung cancer showed significant heterogeneity and evidence of significant bias. The summary sensitivity and specificity was $77.4 \%$ (95\% confidence interval [CI] 65.3-86.1) and 90.1\% (95\% CI 85.3-93.5) when increased uptake against background was used to define fluorodeoxyglucose (FDG) avidity [5]. Patients with FDG-avid mediastinal lymphadenopathy should have lymph node sampling to pathologically confirm that the nodes are involved. Currently, this is most frequently performed by endobronchial ultrasound (EBUS) or other procedures depending on the site of accessible disease. If samples from EBUS are negative for malignancy, the guidelines recommend performing mediastinoscopy before proceeding to surgical resection [6].

Only limited literature is available regarding the diagnostic yield of PET-CT for cervical lymphadenopathy in lung cancer. One study demonstrated that PET-CT was associated with false-positive findings in the neck in 19\% of cases [7]. More studies were performed on the role of PET-CT for the detection of neck lymphadenopathy in head and neck cancer, and within this patient population, PET-CT had a positive predictive value (PPV) of $60-87 \%$ and a specificity of $65-70 \%[8,9]$.

While some guidelines recommend routine NUS in patients with lung cancer and bulky mediastinal lymphadenopathy, the evidence level in the literature is poor and other guidelines do not endorse such a recommendation $[6,10]$. The aim of this study was to investigate the role and diagnostic accuracy of PET-CT compared to NUS for the detection of malignant cervical lymphadenopathy in patients with lung cancer and mediastinal lymphadenopathy on CT.

\section{Materials and Methods}

\section{Study Design and Setting}

This is a retrospective single centre cohort study, based in Galway University Hospital, where, since 2012, the Interventional Respiratory Unit has routinely performed NUS in patients with suspected lung cancer who have bulky mediastinal lymphadenopathy on CT.

\section{Inclusion Criteria}

All patients with confirmed lung cancer and evidence of mediastinal lymphadenopathy on CT defined as a short-axis diameter $>10 \mathrm{~mm}$ who also underwent an NUS in the period between Au- gust 2012 and August 2017 were included. Medical, laboratory and radiology records were reviewed. Procedures performed, pathology results and clinical stage were documented.

Procedures

NUS and Lymph Node Sampling

NUS was performed in a standardised systematic way using a 5- to $12-\mathrm{MHz}$ linear probe with patients in the supine position. Abnormal lymph nodes were defined based on a short-axis diameter of $\geq 5 \mathrm{~mm}$. The fine needle aspiration (FNA) of lymph nodes was performed by 1 of 4 respiratory physicians trained in the procedure. After obtaining informed written consent and using an aseptic technique, local anaesthesia was applied (1-3 mL of 1 or $2 \%$ lidocaine). Between 2 and 4 passes using a 22-gauge needle were performed under direct US guidance. Air-dried and alcoholfixed glass slides were made from the first pass, while the remaining passes were placed in formalin for cell block analysis. If the lymph node was suitable, a core needle biopsy was performed using an 18-gauge SuperCore ${ }^{\mathrm{TM}}$ needle (Aragon Medical Devices, TX, USA). Sampling adequacy was routinely recorded by the pathology department as a quality control measure. Rapid on-site evaluation was not performed for EBUS or NUS FNA.

Positron Emission Tomography-Computerised Tomography

Whole-body PET was performed after the injection of 370-406 MBq FDG with a standard delay time of $90 \mathrm{~min}$. A non-contrast CT from groin to skull base was performed for attenuation correction and diagnostic interpretation. PET-CT was stored and analysed in the Picture Archiving and Communication System (PACS) and reported by 1 of 2 radiologists with more than 10 years' experience. A contrast $\mathrm{CT}$ of the thorax and upper abdomen was done in all patients at baseline and was used for correlation. Findings from PET outside the thorax were documented. The maximum standardised uptake value $\left(\mathrm{SUV}_{\max }\right)$ was routinely reported for all abnormal findings, and a 2.5-cut-off was used to define FDG avidity for cervical lymph nodes.

\section{Outcome Measures and Definitions}

Malignant cervical lymphadenopathy was defined as the presence of malignant cells at cytology or histology consistent with a diagnosis of lung cancer. Diagnostic accuracy of PET was measured against results from NUS and lymph node sampling. Lymph node sampling was considered adequate if lymphoid material or malignant cells were present in the samples.

\section{Statistical Analysis}

Minitab-17 (Minitab ${ }^{\circledR}$ Statistical Software, State College, PA, USA) was used to conduct the statistical analysis. Normally distributed data are described as means (standard deviations), while all other data are described as medians (interquartile ranges) or proportions and 95\% CI. Analysis was performed using MannWhitney $U$ test or Fisher's exact test. A $p$ value of $<0.05$ was considered significant. The sensitivity, specificity, PPV and negative predictive value (NPV) of PET-CT for the detection of cervical lymphadenopathy were calculated.

\section{Ethical Considerations}

This study was approved by the local ethics committee, and patient confidentiality was maintained throughout data collection and analysis. 


\section{Results}

Between August 2012 and August 2017, 223 patients were assessed with NUS in the interventional respiratory unit and 149 patients were diagnosed with lung cancer. Of those, 123 patients had mediastinal lymphadenopathy. Baseline characteristics are shown in Table 1. EBUS was performed in $83 / 123$ patients $(68 \%)$.

\section{NUS and Cervical Lymphadenopathy}

The number of patients with enlarged cervical lymphadenopathy on NUS was 69/123 (56.1\%). The mean (standard deviation) cervical lymph node short diameter was 12.8 (5.9) $\mathrm{mm}$. Needle sampling was technically feasible and therefore performed in 57/123 patients (46.3\%). Findings are summarised in Table 2. Overall, 49/123 patients (39.8\% [95\% CI 31.1-49.1]) had confirmed malignant cervical lymphadenopathy on pathological evaluation. When FNA was compared to core needle biopsy, there was no significant difference in sampling adequacy (94.7 vs. $92.8 \%$; $p=0.80$ ). Immunohistochemistry was possible in $48 / 49$ (98\%) samples. A sample was considered sufficient for molecular analysis when tumour cells represented $>10 \%$ of the population. Molecular analysis was requested in 17 neck lymph node samples and was successfully performed in 14 cases (82.4\% [95\% CI 56.696.2]). There was no significant association between the presence of cervical lymphadenopathy and lung cancer subtype.

\section{PET for Patients with Potentially Curable Disease}

In total, 45/123 (36.6\%) patients with mediastinal lymphadenopathy had potentially curable disease and underwent PET/CT and NUS. FDG-avid cervical lymph nodes were detected in 14/45 (31\%) patients, and following NUS, the number of false positives was 7/14 (50\%). The breakdown of the US findings in this group is shown in Table 3. Overall, PET had a specificity of $81.1 \%$, sensitivity of $87.5 \%$, NPV of $96.8 \%$ and PPV of $50 \%$ for the detection of cervical lymphadenopathy in this group with potentially curable disease. PET did not add any additional staging information in the assessment of the neck region when compared to NUS alone. PET provided additional overall clinical stage information in 4 (8.9\%) patients and led to a change in management in 1 (2.2\%) patient. No relationship was detected between $\mathrm{SUV}_{\max }$ and true- or false-positive PET findings in the neck with a median $S U V_{\max }$ for true-positive cases of 7.0 (IQR 4-11) versus a median $S \mathrm{SV}_{\max }$ for false-positive cases of 3.7 (IQR 3-9; $p=0.11$; Fig. 1). The highest false-
Table 1. Baseline characteristics of 123 patients with confirmed lung cancer and mediastinal lymphadenopathy that underwent a neck ultrasound

$\begin{array}{lc}\text { Gender } & \\ \quad \text { Male } & 70(56.9) \\ \text { Female } & 53(43.1) \\ \text { Age, years } & 67.4 \pm 11.2 \\ \text { Small cell lung cancer } & 27(22) \\ \text { Non-small cell lung cancer } & 96(78) \\ \quad \text { Adenocarcinoma } & 56(45.5) \\ \text { Squamous cell carcinoma }_{\text {Other }^{\mathrm{a}}} & 32(26) \\ \end{array}$

Data are shown as $n(\%)$ or means \pm standard deviations. a "Other" includes neuroendocrine and poorly differentiated lung cancer.

Table 2. Results of NUS and needle sampling of 57 patients with suspicious cervical lymphadenopathy on NUS

\begin{tabular}{lc}
\hline Short diameter, cm & $12.8 \pm 5.9$ \\
Enlarged lymph nodes & $69(56.1)$ \\
Needle sampling & $57(46.3)$ \\
$\quad$ FNA alone & $43(35)$ \\
FNA and CNB & $14(11.4)$ \\
Sampling adequacy, \% & \\
FNA & 94.7 \\
CNB & 92.8 \\
Malignant cervical lymphadenopathy & $49(39.8)$ \\
Small cell lung cancer & $13(26.5)$ \\
Adenocarcinoma & $20(40.8)$ \\
Squamous cell carcinoma & $12(24.5)$ \\
Other & $4(8.2)$ \\
\hline
\end{tabular}

Data are reported as $n(\%)$ or means \pm standard deviations, unless otherwise stated. NUS, neck ultrasound; FNA, fine needle aspiration; CNB, core needle biopsy.

positive FDG uptake was due to lymphoma which was evident on cytology and confirmed later by surgical excision.

\section{Discussion}

In this cohort, $39.8 \%$ of patients with lung cancer and mediastinal lymphadenopathy had confirmed malignant cervical lymphadenopathy following NUS. These findings are consistent with the results reported by Kumaran et al. [4] who investigated the role of routine NUS in 101 patients with suspected and later confirmed lung cancer 
Table 3. Results of PET, NUS and cytology in all 7 patients that were considered to have false-positive PET findings in the neck

\begin{tabular}{|c|c|c|c|c|}
\hline Case & $\mathrm{SUV}_{\max }$ & NUS findings & LN short diameter, $\mathrm{mm}^{\mathrm{a}}$ & Cytology findings \\
\hline 1 & 17.7 & bilateral cervical LNs & 14 & lymphoma \\
\hline 2 & 3 & no lymphadenopathy & & \\
\hline 3 & 4.2 & left cervical LN & 10 & non-specific lymphadenitis \\
\hline 4 & 5 & no lymphadenopathy & & \\
\hline 5 & 4 & no lymphadenopathy & & \\
\hline 6 & 3 & no lymphadenopathy & & \\
\hline 7 & 3.4 & bilateral enlarged LNs & 12 & lymphoma \\
\hline
\end{tabular}

Two cases of lymphoma on cytology were later confirmed on surgical excisional biopsy. PET, positron emission tomography; NUS, neck ultrasound; $\mathrm{SUV}_{\max }$, maximum standardised uptake value; LN, lymph node. ${ }^{a} \mathrm{LN}$ short diameter is reported for the largest $\mathrm{LN}$ as measured on US.

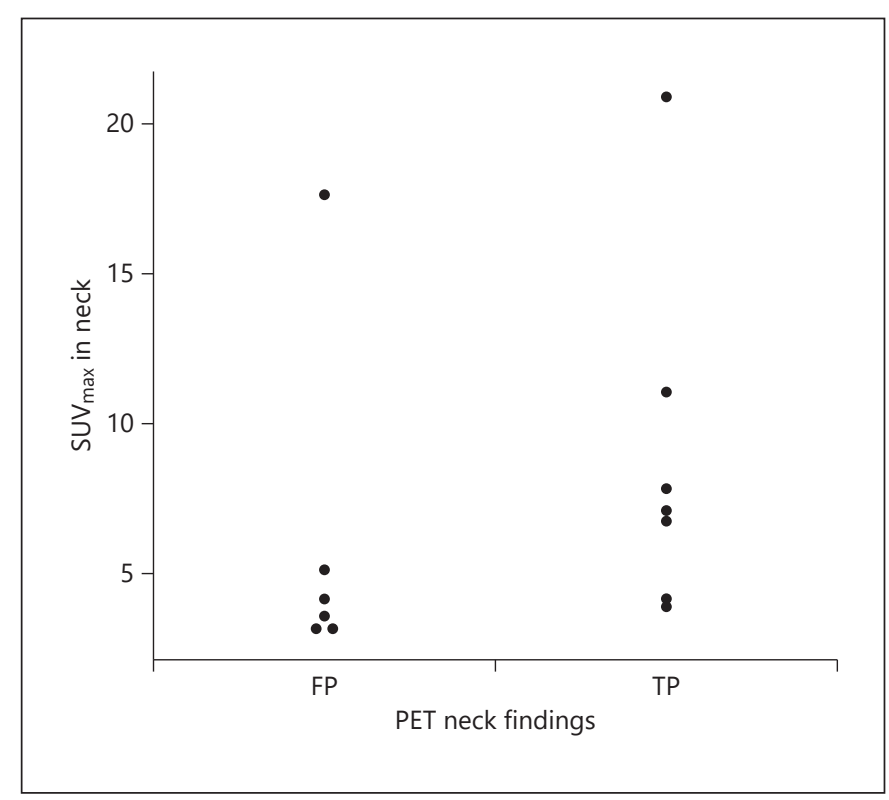

Fig. 1. Comparison of FDG uptake in patients with true- and falsepositive findings in the neck on PET-CT. $\mathrm{SUV}_{\max }$, maximum standardised uptake value; FP, false positive; TP, true positive; FDG, fluorodeoxyglucose; PET, positron emission tomography; CT, computed tomography.

that had evidence of mediastinal lymphadenopathy. The authors reported that $50 \%$ of patients had malignant neck lymph nodes. Similarly, a study of 40 patients with nonpalpable neck lymph nodes confirmed that $40 \%$ had supraclavicular lymph nodes metastases [11].

The presence of supraclavicular lymphadenopathy in non-small cell lung cancer indicates $\mathrm{N} 3$ disease consistent

PET and NUS for the Detection of Cervical Lymphadenopathy with at least stage IIIB [12]. Such cN3 disease is associated with a median survival of 7 months in patients with non-small cell lung cancer [13]. The National Institute of Health and Care Excellence (NICE) guidelines recommend routine NUS in patients with bulky mediastinal lymphadenopathy or blind transbronchial needle aspiration mostly based on a cost-effectiveness analysis, although the level of evidence is weak [10]. The findings from our study support this recommendation - potentially identifying nodal disease in the neck region in about $40 \%$ of patients. This can significantly streamline investigations and prevent the need for more invasive tests, potentially reducing futile treatments and time to definitive diagnosis and clinical stage. If these findings are supported by further well-designed prospective studies of routine NUS in patients with mediastinal lymphadenopathy, they will have a significant impact on the diagnostic and staging pathway in lung cancer considering the feasibility and safety of performing NUS even in the office.

While PET had a high NPV for the detection of malignant cervical lymphadenopathy, it was associated with a high rate of false-positive findings. The diagnostic accuracy of PET for malignant neck lymphadenopathy in lung cancer has previously been studied; Sung et al. [7] reported on 32 patients with cervical lymphadenopathy detected on PET and/or CT and found a false-positive rate of $19 \%$. The authors reported an $\mathrm{SUV}_{\max }$ of 2.3 to be the optimum cut-off for differentiating true- and false-positive findings. A smaller study of 12 patients with lung cancer and FDG-avid cervical lymphadenopathy showed supraclavicular metastases from lung cancer in 10 cases; 1 case showed evidence of sarcoidosis and another evidence of metastatic breast cancer [14]. Mean $S U V_{\max }$ in 
this study was 6.3 (range 3.7-12.2). In our study, NUS was performed by respiratory physicians trained in the procedure and, while it can be argued that there could have been a failure to detect some malignant neck lymphadenopathy, the high sampling adequacy rate of $94.7 \%$ in this cohort would be inconsistent with such an argument.

In this paper, we report that PET offered no additional staging value in the neck when NUS was also performed in this cohort. In addition, it only changed $\mathrm{M}$ stage in $8.8 \%$ of patients from finding disease in other sites, which led to a change in management in only $2.2 \%$ of cases. Similar results were seen in a multicentre retrospective study of 1,059 patients who underwent PET scanning for suspected lung cancer [15]. In this population, additional extra-thoracic findings were detected in $15.8 \%$, but management changed in only $1.2 \%$ of all patients that underwent PET. The results from this study and our study suggest that PET/CT has only a limited additional staging value once NUS is performed and the mediastinum is properly staged. However, a recommendation regarding the benefit of PET when NUS is negative cannot be made based on this study as the sample size of this subgroup was small ( 38 patients).

This study is limited by its retrospective design and the possibility of selection bias. Only patients that underwent an NUS were assessed for inclusion in the study, which might have led to confounding by indication bias. Also, this is a single centre study, which might affect the generalisability of its findings.

\section{Conclusions}

In this cohort of patients with lung cancer and mediastinal lymphadenopathy, routine NUS performed by respiratory physicians revealed malignant cervical lymphadenopathy in $39.8 \%$ of cases. Once NUS was performed, PET did not add any staging value in the neck and only limited additional staging value elsewhere with change in management in just $2.2 \%$ of cases. Further prospective studies in this subgroup of patients with suspected lung cancer is required to further assess the role of PET in the staging algorithm once NUS is incorporated as a routine investigation.

\section{Acknowledgements}

Dr Mohammed Ahmed acknowledges the receipt of support from the European Respiratory Society (ERS fellowship ID STRTF 2016-7508).

\section{Disclosure Statement}

The authors declare no competing interests.

\section{References}

1 Meguid RA, Hooker CM, Harris J, Xu L, Westra WH, Sherwood JT, et al: Long-term survival outcomes by smoking status in surgical and nonsurgical patients with non-small cell lung cancer: comparing never smokers and current smokers. Chest 2010;138:500-509.

2 Asamura H, Chansky K, Crowley J, Goldstraw P, Rusch VW, Vansteenkiste JF, et al: The International Association for the Study of Lung Cancer Lung Cancer Staging Project: proposals for the revision of the $\mathrm{N}$ descriptors in the forthcoming 8th edition of the TNM classification for lung cancer. J Thorac Oncol 2015;10:1675-1684.

3 Kendirlinan R, Ozkan G, Bayram M, Bakan ND, Tutar M, Gür A, et al: Ultrasound guided fine-needle aspiration biopsy of metastases in nonpalpable supraclavicular lymph nodes in lung cancer patients. Multidiscip Respir Med 2011;6:220-225.
4 Kumaran M, Benamore RE, Vaidhyanath R, Muller S, Richards CJ, Peake MD, et al: Ultrasound guided cytological aspiration of supraclavicular lymph nodes in patients with suspected lung cancer. Thorax 2005;60:229-233.

5 Schmidt-Hansen M, Baldwin DR, Hasler E, Zamora J, Abraira V, Roqué I, Figuls M: PETCT for assessing mediastinal lymph node involvement in patients with suspected resectable non-small cell lung cancer. Cochrane Database Syst Rev 2014;CD009519.

6 Detterbeck FC, Lewis SZ, Diekemper R, Addrizzo-Harris D, Alberts WM: Executive summary: diagnosis and management of lung cancer, 3rd ed: American College of Chest Physicians evidence-based clinical practice guidelines. Chest 2013;143(5 suppl):7S-37S.

7 Sung YM, Lee KS, Kim B-T, Kim S, Kwon OJ, Choi JY, et al: Nonpalpable supraclavicular lymph nodes in lung cancer patients: preoperative characterization with 18 F-FDG PET/ CT. AJR Am J Roentgenol 2008;190:246-252.
8 Nakamura S, Okochi K, Kurabayashi T: Dualtime-point fluorodeoxyglucose positron emission tomography for diagnosis of cervical lymph node metastases in patients with head and neck squamous cell carcinoma. J Comput Assist Tomogr 2011;35:303-307.

9 Carlson ER, Schaefferkoetter J, Townsend D, McCoy JM, Campbell PD, Long M: The use of multiple time point dynamic positron emission tomography/computed tomography in patients with oral/head and neck cancer does not predictably identify metastatic cervical lymph nodes. J Oral Maxillofac Surg 2013;71: 162-177.

10 National Institute for Health and Care Excellence (NICE): Lung cancer: diagnosis and management. Guidance and guidelines. https://www.nice.org.uk/guidance/cg121 (accessed June 26, 2017). 
11 Ozkan G, Tutar M, Bayram M, Bakan D, Gür A, Camsari G: The impact of ultrasonography-guided fine needle aspiration of no palpable supraclavicular lymph nodes on diagnosis and staging in advanced lung cancer. Tuberk Toraks 2009;57:186-191.

12 Goldstraw P, Chansky K, Crowley J, RamiPorta R, Asamura H, Eberhardt WEE, et al: The IASLC Lung Cancer Staging Project: proposals for revision of the TNM stage groupings in the forthcoming (eighth) edition of the TNM classification for lung cancer. J Thorac Oncol 2016;11:39-51.
13 Groome PA, Bolejack V, Crowley JJ, Kennedy C, Krasnik M, Sobin LH, et al: The IASLC Lung Cancer Staging Project: validation of the proposals for revision of the $\mathrm{T}, \mathrm{N}$, and $\mathrm{M}$ descriptors and consequent stage groupings in the forthcoming (seventh) edition of the TNM classification of malignant tumours. J Thorac Oncol 2007;2:694-705.
14 Werner L, Keller FA, Bhure U, Roos JE, Tornquist K, del Sol Pèrez-Lago M, et al: The value of ultrasound-guided biopsy of fluorodeoxyglucose positron emission tomography (FDG-PET)-positive supraclavicular lymph nodes in patients with suspected lung cancer. BMC Med Imaging 2017;17:41.

15 Arens AIJ, Postema JWA, Schreurs WMJ, Lafeber A, Hendrickx BW, Oyen WJG, et al: FDG-PET/CT limited to the thorax and upper abdomen for staging and management of lung cancer. PLoS One 2016;11:e0160539. 